\title{
Strain-Induced Crystallization, Part III: Theory
}

\author{
G. S. Y. YEH and K. Z. HONG \\ Departments of Chemical Engineering and Materials \& Metallurgical Engineering \\ and Macromolecular Research Center \\ University of Michigan \\ Ann Arbor, Michigan 48109
}

\begin{abstract}
A nucleation theory for strain-induced crystallization is formulated to explain and to predict the effects of molecular strain on crystallization kinetics and crystallite size. Unlike any current theories that have based their formulations on some assumed extended-chain line nuclei or folded-chain crystals, the present theory avoids all assumptions concerning the crystal morphology. It is based on experimental findings which indicate limited crystal growth in the strain direction, following a reciprocal dependence of crystal thickness on supercooling $\Delta T .\left(\Delta T=T_{m}^{o}-T\right.$, where the equilibrium melting temperature, $T_{m}^{o}$, is a variable dependent on degree of molecular strain prior to strain-induced crystallization.) It is predicted that the logarithm of the nucleation rate, $N^{o}$, is dependent on $\left(T_{m}^{o}\right)^{2} / T(\Delta T)^{2}$ or $T_{m}^{o} / T(\Delta T)$, and that the critical nucleus thickness $l^{*}$ is shown to be proportional to $T_{m}^{o} / \Delta T$. In addition, expressions are also presented, including examples, to show the dependence of $N^{o}, l^{* o}$ and $T_{m}^{o}$ on degree of molecular strain, $\epsilon$, or melt entropy reduction, $\Delta s^{\prime}$. Our analysis predicts that, on comparing a polyethylene crystallized in the presence of strain to one crystallized in the absence of strain at $130^{\circ} \mathrm{C}$, an increase in "coil" dimension of less than about 50 percent can bring about a $10^{4}$ fold increase in heterogeneous nucleation rate, a 30-40 percent reduction in critical nucleus thickness and a $10^{\circ} \mathrm{C}$ increase in equilibrium melting temperature. These results will be discussed and compared with available experimental evidence.
\end{abstract}

\section{INTRODUCTION}

$\mathrm{T}$ he fabrication of crystallizable polymers usually involves melt flow and some degree of molecular strain, and therefore in many instances with concomitant stress or strain-induced crystallization (SIC) (1). For example, SIC occurs during processing of monofilaments, oriented films or various shaped products during mold filling. Hence, ever since this phenomenon was discovered in 1805 (2), the elucidation of its crystal morphology, kinetics and properties has been of considerable interest both technologically and scientifically. Therefore, we were not too surprised to find that one of the earliest applications of X-ray diffraction was on SIC of "rubberlike" polymers; the results were published by Katz in 1925 (3). Nor were we surprised to learn one of Flory's many contributions to polymer science to be also on SIC (4). It dealt with the associated stress relaxation phenomenon which occurs during crystallization and the elevation of equilibrium melting point. Since these classical publications, other similar studies on SIC crystallites, crystallization kinetics (in terms of stress relaxation) and equilibrium melting point elevations have also appeared, especially in the past 10-15 years. The following are some of the more significant findings that were reviewed in Parts I and II (1).
1. The gross morphology of SIC tends to be either "fibrillar" or "lamellar."

2. The basic crystalline units which give rise to either the fibrillar or the lamellar morphology have limited thicknesses and lateral dimensions $(\sim 100-300 \AA)$. It is the degree of their molecular $c$-axis orientation and their lateral correlation with neighboring crystallites, which determines whether the observed gross morphology is fibrillar, well-oriented or twisted lamellar.

3. The primary crystallization of SIC occurs very fast. It is usually completed within a few seconds. Additional crystallization and/or crystallite rearrangement can occur under strain during stress relaxation or stress rise.

4. Formation of chain-folded lamellae occurs during stress relaxation. Stress changes during crystallization under strain do not reflect changes in chain conformation, but can reflect morphological rearrangements under constant strain.

5. Melting point elevation can be as much as 50$100^{\circ} \mathrm{C}$ above the isotropic equilibrium melting point of a polymer.

These findings are typical of most polymers whether they are rubbers $(5-9)$, glasses $(10,11)$ or highly crystallizable polymers like polyethylene, PE $(12,13)$; and, as pointed out in Parts I and II, they are consistent with a structural model being formulated by us since 1967 , but 
not with the theoretical extended-chain line nucleus model proposed by Keller and Machin (14). Both models (Fig. 1) show the presence of oriented "fibrils" and "lamellae" in strain-crystallized polymers, although their structure and origin, as discussed before (1), differ substantially and can have very different consequences. For example, the extended-chain line nucleation hypothesis has led to several proposed mechanisms (15-17) for the fibril formation, each of which is based on the possibility that fully extended-chain crystals are formed during SIC. It has also led to numerous attempts in the past decade to generate extended-chain crystals (ECC) for high modulus fibers by means of SIC, but so far have met little success. This we now learn is primarily due to the fact that SIC does not directly lead to extended-chain crystals; only through additional deformation, either after (12) and/or during (18) the formation of shish-kabob structure, can one expect the formation of highly extended-chain crystals and high modulus fibers. Most of the observed increase in modulus in straincrystallized polymers can be accounted for without having to invoke the extended-chain crystal hypothesis as was recently demonstrated by Kapuscinski, Ward and Scanlan (19). Nevertheless the extended-chain line nucleus hypothesis is a rather appealing model; it has often led Keller and co-workers to invoke observed melting point elevations as proofs for the presence of ECC (20), not realizing that these elevations can be theoretically accounted for by the entropy decreases in oriented melts as was done by Flory nearly 30 years ago (4). Otherwise it would be very difficult to explain why melting point elevations can reach as high as $50-100^{\circ} \mathrm{C}$ above the normal equilibrium melting points, i.e., above the melting points for extended-chain crystals (1).

Another major consequence of Keller's model has to do with the assumption that oriented chain-folded lamellae crystallize in the usual fashion, i.e., like crys-

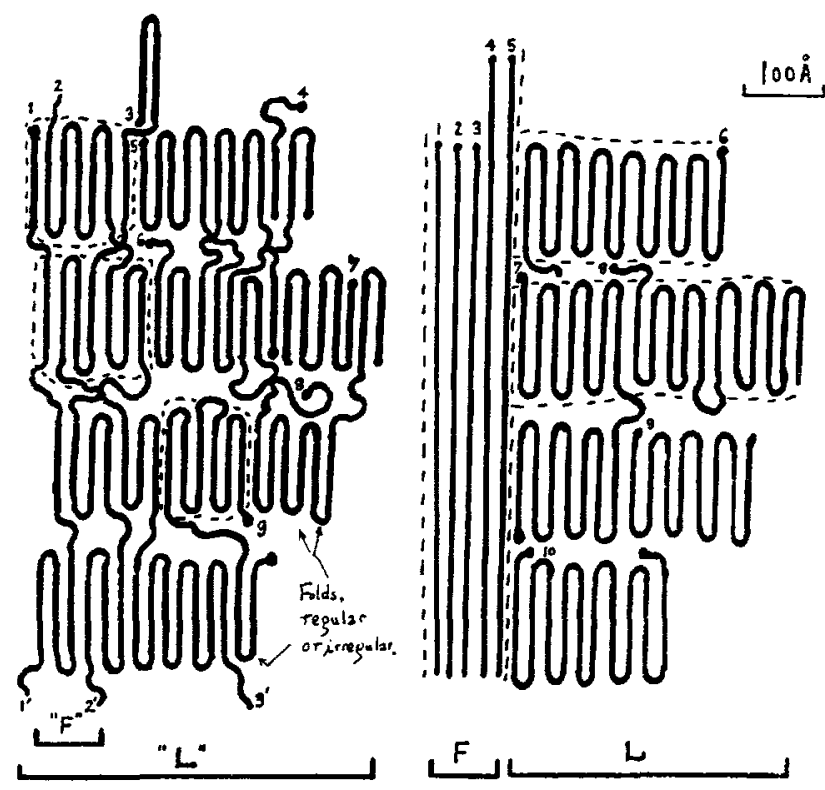

Fig. 1. Models depicting strain-induced crystallization mechanisms of polymers, left: Yeh, et al. (5-13) and right: Keller and Machin (14). The dotted lines around the crystallites emphasize the differences between the two models. tallization in the quiescent state, except that they nucleate epitaxially onto the supposedly extended-chain line nuclei. This would mean that the lamellar thickness should be dependent on crystallization temperature only, not on strains or strain rates. This is also not consistent with experimental findings, including results obtained in their own laboratories (21). They often indicate that in addition to the usual dependency on crystallization temperature, observed long periods $L_{o b s}^{o}$ or observed crystal thicknesses $l_{\text {obs }}^{o}$ depend also on strains or strain rates (e.g., natural rubber $(5,6)$, transpolyisoprene $(8,9)$, PE (22), and stirrer crystallized PE (21)), showing a decrease with increased strains or strain rates. Such dependency suggests that $L_{o b s}^{o}$ or $l_{o b s}^{o}$ must be directly related to the critical nucleus thickness $l^{o *}$, since $l^{0 *}$ is expected to decrease with increasing strains or strain rates on account of the $T_{m}$ elevation due to the imposed strain or strain rates and to follow an inverse relationship with supercooling $\Delta T$. Therefore at a constant crystallization temperature $l^{o *}<l^{*}\left(l^{*}\right.$ being the critical nucleus formed in the quiescent state) and that the thickness of SIC crystallites, like that in the quiescent crystallization $(Q C)$ case, is also limited. However, we believe that the observed limited growth in SIC crystallite thickness along the stretch direction stems from a very different origin than the ones proposed by Lauritzen and Hoffman for QC (23) or by Kobayashi and Nagasawa (24) for SIC, both assuming chain folding occurring during crystallization. We believe it is caused by defects along the chain segments in the form of folds originally present in the amorphous state (25). They impede growth along the $c$-axis direction during crystallization.

Thus, the observed relation between $l_{o b s}^{o}\left(\right.$ or $\left.L_{o b s}^{o}\right)$ and $l^{* o}$ suggests that a classical type nucleation theory can also be formulated for SIC without introducing any assumptions concerning the SIC chain conformation or crystal morphology, since in both instances (QC or SIC), polymer molecules crystallize essentially from the same kind of melt or glassy structures with folds already being present. The surfaces and therefore the end $\left(\sigma_{e}\right)$ and side $\left(\sigma_{s}\right)$ surface free energies for a SIC critical nucleus can be considered approximately the same as those for a QC critical nucleus. Such a theory will be introduced here in Part III. Part IV (26) discusses the application of theory to the analysis of nucleation rates in terms of induction times of shear-crystallized polyethylene and the prediction of changes in long periods and melting point elevations from nucleation rates. Part V (27) will introduce a new equation which gives a more accurate prediction for the equilibrium melting behavior of strain-crystallized polymers (crosslinked or uncrosslinked) than the ones obtained by Flory (4) or Krigbaum and Roe (28).

\section{THERMODYNAMIC DRIVING FORCE FOR STRAIN-INDUCED CRYSTALLIZATION, $\Delta \mathbf{f}^{o}$}

As in the case of classical nucleation theory that was developed for any condensed systems by Turnbull and Fisher (29) the fundamental driving force for nucleation of polymers in the quiescent state $(23,30)$ is $\Delta f$ (difference in free energies between melt and crystal) and for 
nucleation in the oriented state is $\Delta f^{\circ}$ (difference in free energies between oriented melt and crystal). Since $\Delta f=$ $\Delta H-T \Delta S$ and $\Delta f^{o}=\Delta H^{o}-T \Delta S^{\circ}$ where $\Delta H$ and $\Delta S$, and $\Delta H^{o}$ and $\Delta S^{\circ}$ are the corresponding differences in enthalpy of fusion and entropy of fusion, the difference in the driving force between quiescent crystallization (QC) and strain-induced crystallization (SIC) is $\Delta f^{o}-\Delta f$ $=\left(\Delta H^{o}-\Delta H\right)-T\left(\Delta S^{o}-\Delta S\right)$. The difference, $\Delta f^{o}-\Delta f$, is then the difference in free energy between macromolecules in an oriented "coiled" state and the unoriented "coiled" state. This difference is essentially $-T\left(\Delta S^{\circ}-\Delta S\right)$ or $T \Delta S^{\prime}$ since the difference, $\Delta H^{0}-\Delta H$, is about 3 orders of magnitude smaller than $\Delta S^{\prime}(31)$ and as expected based on rubber elasticity theory. Consequently $\Delta f^{\circ}$ can be expressed as $\Delta f+T \Delta S^{\prime}$, and the free energy of driving force for SIC nucleation is therefore raised by $T \Delta S^{\prime}, \Delta S^{\prime}$ being positive. $\Delta S^{\prime}$ can be considered as a measure of degree of molecular strain, $\epsilon$, in the "structure" state just prior to SIC and will be shown to be directly proportional to $\epsilon$ later.

\section{NUCLEATION RATES AND ENHANCEMENT OF NUCLEATION RATES, $N^{\circ} / \mathbf{N}$}

Again, following Turnbull and Fisher we can express the nucleation rate per unit volume and time as $N=N_{c}$ $\exp -E_{D} / k T_{\text {exp }}-\Delta F * / k T$. Here, all the constants have the usual meanings as in the case of QC of polymers (30). $E_{D}$ is the activation energy for transport, $N_{c}$ is the front factor, $\Delta F^{*}$ is the free energy for the formation of nucleus of critical dimensions ( $\nu a^{*}$ and $\left.l^{*}\right), k$ is Boltzmann's constant and $T$ is the crystallization temperature. $\Delta F^{*}$ depends on the geometry of the nucleus and on the nucleation process. If we now follow Price (30), $\Delta F^{*}$ $(\mathrm{QC})$ and $\Delta F^{* o}$ can be derived without making any assumptions about the critical nucleus chain conformation (fringed-micelle or chain-folding). For polymers crystallized by means of a homogeneous and threedimensional nucleation process in the quiescent state, $\Delta F^{*}=A \sigma_{s}^{2} \sigma_{e} /(\Delta f)^{2}$ and in the strained rate, $\Delta F^{* 0}=$ $A\left(\sigma_{s}^{o}\right)^{2} \sigma_{e}^{o} /\left(\Delta f+T \Delta S^{\prime}\right)^{2}$. For a heterogeneous nucleation process, $\Delta F^{*}=B \sigma_{e} \sigma_{s} / \Delta f$ for $Q C$ and $\Delta F^{* 0}=B \sigma_{e}^{o} \sigma_{s}^{o} /(\Delta f$ $\left.+T \Delta S^{\prime}\right)$ for SIC, where $A$ and $B$ are geometrical constants which depend on the shape of the nucleus, $\sigma_{s}, \sigma_{s}^{o}$ and $\sigma_{e}$, $\sigma^{0}{ }_{e}$ are the well-known corresponding lateral interfacial free energies and the end surface free energies per unit area. All other energies, such as edge free energy, including the free energy difference arising between the meltsubstrate and the crystal-substrate are considered negligible in the derivations.

If we assume $\sigma_{s}^{o}$ and $\sigma_{e}^{o}$ to have the same values as $\sigma_{s}$ and $\sigma_{e}$, based on the fact that polymers crystallize essentially from the same "structure" state with prefolds (25), one can write down the following corresponding equations for homogeneous nucleation and show that the changes in nucleation rate are primarily due to $\Delta f^{o}$ as in the QC case:

$$
\begin{aligned}
N & =N_{c} \exp \left[-E_{D} / k T\right] \exp \left[-A \sigma_{s}^{2} \sigma_{e} /(\Delta f)^{2} k T\right] \\
N^{o} & =N_{c} \exp \left[-E_{D} / k T\right] \exp \left[-A \sigma_{s}^{2} \sigma_{e} /\left(\Delta f^{o}\right)^{2} k T\right]
\end{aligned}
$$

and for heterogeneous nucleation:

$$
\begin{aligned}
& N=N_{c} \exp \left[-E_{D} / k T\right] \exp \left[-B \sigma_{s} \sigma_{e} / \Delta f^{o}(k T)\right] \\
& N^{o}=N_{c} \exp \left[-E_{D} / k T\right] \exp \left[-B \sigma_{s} \sigma_{e} / \Delta f^{o}(k T)\right]
\end{aligned}
$$

With the usual assumptions that $\Delta f \cong(\Delta H)(\Delta T) / T_{m}$ and $\Delta f^{o} \cong \Delta H(\Delta T) / T_{m}^{o}$ where $T_{m}$ and $T_{m}^{o}$ are the corresponding equilibrium melting temperatures for QC and SIC, and $\Delta T$, the corresponding degrees of supercooling, one can rewrite Eqs 1-4 in terms of the usual $\Delta T$ effects on the nucleation rates as follows:

$$
\begin{aligned}
& N=N_{c} \exp \left(-E_{D} / k T\right) \\
& \exp \left[-A \sigma_{s}^{2} \sigma_{e} T_{m}^{2} /(\Delta H)^{2} k T(\Delta T)^{2}\right] \\
& N^{o}=N_{c} \exp \left(-E_{D} / k T\right) \\
& \exp \left[-A \sigma_{s}^{2} \sigma_{e}\left(T_{m}^{o}\right)^{2} /(\Delta H)^{2} k T(\Delta T)^{2}\right] \\
& N=N_{c} \exp \left(-E_{D} / k T\right) \\
& \exp \left[-B \sigma_{s} \sigma_{e} T_{m} / \Delta H k T(\Delta T)\right] \\
& N^{0}=N_{c} \exp \left(-E_{D} / k T\right) \\
& \exp \left[-B \sigma_{s} \sigma_{e} T_{m}^{o} /(\Delta H) k T(\Delta T)\right]
\end{aligned}
$$

Based on the fact that the diffusion transport is negligible in the high temperature region, the enhancements of nucleation rates in terms of $N^{\circ} / \mathrm{N}$ can be expressed as follows:

- homogeneous nucleation

$$
\frac{N^{o}}{N}=\exp \frac{A \sigma_{s}^{2} \sigma_{e}}{k T}\left[\frac{1}{(\Delta f)^{2}}-\frac{1}{\left(\Delta f+T \Delta S^{\prime}\right)^{2}}\right]
$$

- heterogeneous nucleation

$$
\frac{N^{o}}{N}=\exp \frac{B \sigma_{s} \sigma_{e}}{k T}\left[\frac{1}{\Delta f}-\frac{1}{\Delta f+T \Delta S^{\prime}}\right]
$$

One can readily see that the nucleation rates can be greatly enhanced upon melt orientation and the degree of enhancement $N^{\%} / \mathrm{N}$ can be expressed as a function of $\Delta S^{\prime}$, the reduction in melt entropy.

\section{DIMENSIONS OF CRITICAL NUCLEUS, $\nu a^{* o}$ AND $l^{* o}$}

Following Price (30), the lateral area, $\nu a^{* 0}$, and the longitudinal thickness, $l^{* 0}$, of a critical nucleus for SIC can be similarly derived as:

$$
\begin{gathered}
\nu a^{* o}=\frac{K_{1}\left(\sigma_{s}^{o}\right)^{2}}{\left(\Delta f^{o}\right)^{2}}=\frac{K_{1} \sigma_{s}^{2}}{\left(\Delta f+T \Delta S^{\prime}\right)^{2}} \\
l^{* o}=\frac{K_{2} \sigma_{e}^{o}}{\Delta f^{o}}=\frac{K_{2} \sigma_{e}}{\left(\Delta f+T \Delta S^{\prime}\right)}
\end{gathered}
$$

where $K_{1}$ and $K_{2}$ are constants. For example, $K_{2}=4$ or 2 , depending on the nucleation process whether it be homogeneous or heterogeneous. Consequently, according to Eqs 11 and $12, \nu a^{* o}(\mathrm{SIC})<\nu a^{*}(\mathrm{QC})$ and $l^{* o}(\mathrm{SIC})$ $<l^{*}(\mathrm{QC})$. In terms of the usual $\Delta T$ effects,

$$
\begin{aligned}
l^{* o} & =\frac{4 \sigma_{e} T_{m}^{o}}{\Delta H \Delta T} \text { (homogeneous) } \\
l^{* o} & =\frac{2 \sigma_{e} T_{m}^{o}}{\Delta H \Delta T} \text { (heterogeneous) }
\end{aligned}
$$

$T_{m}^{o}$ is now a variable, dependent on $\Delta S^{\prime}$. For 
heterogeneous nucleation, an additional term (e.g., $\delta l$ (32)) must be added to $l^{* o}$ for stable crystal growth. Therefore observed $l_{o b s}^{o}$ should be somewhere between $l^{* 0}$ (heterogeneous) and $l^{* 0}$ (homogeneous).

\section{OBSERVED MELTING TEMPERATURE, $T_{o b s}^{o}$, AND EQUILIBRIUM MELTING TEMPERATURE, $T_{m}^{o}$}

The observed melting temperature should show, as in the case of QC, a melting point depression due to limited crystal thicknesses. It can be similarly expressed as

$$
T_{o b s}^{o}=T_{m}^{o}\left(1-\frac{2 \sigma_{e}}{\Delta H} \frac{1}{l_{o b s}^{o}}\right)
$$

The equilibrium melting temperature $T_{m}^{o}$ for SIC is given by

$$
T_{m}^{o}=\frac{\Delta H^{o}}{\Delta S^{o}}=\frac{\Delta H}{\Delta S-\Delta S^{\prime}}
$$

or

$$
\frac{1}{T_{m}^{o}}=\frac{\Delta S}{\Delta H}-\frac{\Delta S^{\prime}}{\Delta H}=\frac{1}{T_{m}}-\frac{\Delta S^{\prime}}{\Delta H}
$$

where $T_{m}$ is the equilibrium melting temperature for QC. $\Delta S^{\prime}$ is therefore the well-known source of straininduced equilibrium melting temperature elevation and is the same thermodynamic parameter that controls the increases in SIC nucleation rates (Eqs 9 and 10 ) or the decreases in SIC critical nucleus dimensions (Eqs 11 and 12).

\section{RELATION BETWEEN $\triangle S^{\prime}$ AND MOLECULAR EXTENSION RATIO, $\alpha$, PRIOR TO SIC}

According to Flory (4), Krigbaum and Roe (28), and Hong and Yeh (27), $\Delta S^{\prime}$ and $\alpha$ are related as follows:

$\Delta S_{1}^{\prime}=\frac{\mathrm{k} \tilde{N}}{2}\left[\left(\frac{24 m}{\pi}\right)^{1 / 2}\right.$

$$
\left.\alpha-\left(\alpha^{2}+\frac{2}{\alpha}\right)\right] \text { (Flory) }
$$

$\Delta S_{2}^{\prime}=\frac{\mathrm{k} \tilde{N}}{2}\left(\alpha^{2}+\frac{2}{\alpha}-3\right) \quad$ (Krigbaum-Roe)

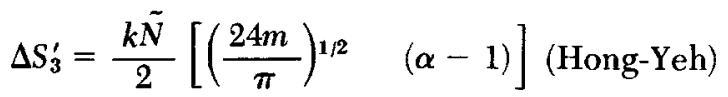

$$
=\frac{\mathrm{kN}}{2}\left(\frac{24 m}{\pi}\right)^{1 / 2} \epsilon
$$

If affine deformation applies, then $\alpha$ can be equated to the macroscopic stretch ratio. $m$ is the number of statistical segments per "network" chain and $N$ is the number of "network" chains per unit volume.

Equations 17 or $17^{\prime}$ is introduced because it is a well-known established fact that neither $E q 15$ nor 16 fits the experimental data. Krigbaum and Roe's equation, which is based on rubber elasticity theory, predicts a constantly lower $T_{m}^{o}$ than reported values, suggesting that the strained melt which leads to SIC is not amenable to analysis based on rubber elasticity theory. Flory's equation gives an overestimation at $\alpha$ close to 1 and an underestimation at $\alpha>5$. Based on careful analyses of all the available $T_{m}^{o}$ data reported by several investigators (38-41), Hong and Yeh have found (27) that $1 / T_{m}^{o}$ decreases linearly with $\alpha-1$ and the slope is surprisingly close to the coefficient of $\alpha$ in Flory's equation. They thus were able to establish an empirical equation describing the anisotropic $T_{m}^{o}$ as a function of $\alpha$ and isotropic $T_{m}$. Equation $17^{\prime}$, which shows a direct dependency of $\Delta S^{\prime}$ on molecular strain, was derived from their empirical equation (27). It fits data better than either $E q s 15$ or 16 and can be used to relate $\Delta S^{\prime}$ to other measurable orientation parameters such as birefringence.

\section{THEORETICAL PREDICTIONS OF $N^{\circ} / N, l^{o *}$ AND $T_{m}^{o}$}

As examples of how the enhancement of nucleation rate $N^{\sigma} / N$, critical nucleus thickness $l^{* o}$ and equilibrium melting temperature $T_{m}^{o}$ depend on molecular extension $\alpha$ or the structure state $\Delta S^{\prime}$, theoretical plots are presented in Figs. 2 and 3 for a polyethylene crystallized at $130^{\circ} \mathrm{C}$, assuming two different nucleation processes. The constants used for polyethylene are (33) $A=8 \pi, B$ $=4 b_{o}\left(b_{o}=4.11 \AA\right), T_{m}=145^{\circ} \mathrm{C}, \Delta H=2.8 \times 10^{9}$ ergs $/ \mathrm{cm}^{3}, \sigma_{s}=10 \mathrm{ergs} / \mathrm{cm}^{2}, \sigma_{e}=80 \mathrm{ergs} / \mathrm{cm}^{2}, \tilde{N}=1.5 \times$ $10^{20}$ "network" chains $/ \mathrm{cm}^{3}$ (assuming the molecular weight between chain entanglements for polyethylene

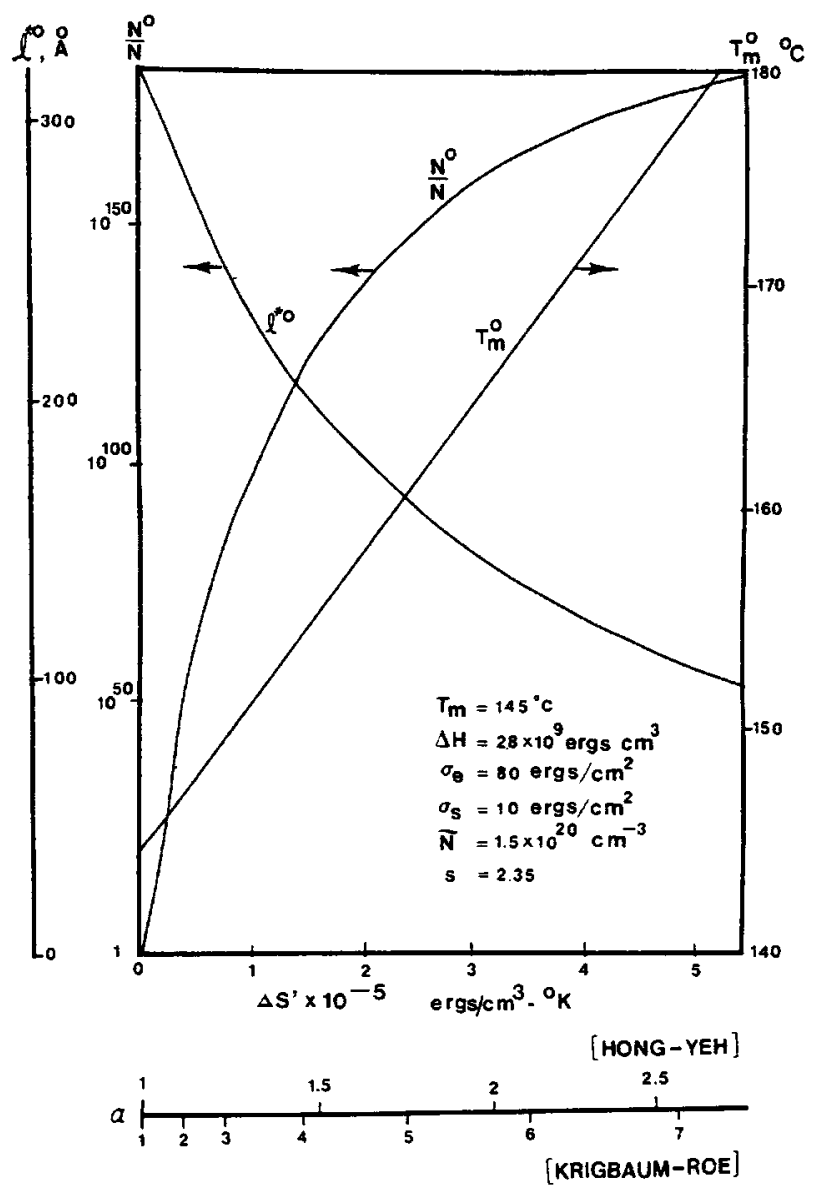

Fig. 2. Theoretical plots of $N^{\circ} / N, l^{* o}$ and $T_{m}^{o}$ vs $\Delta S^{\prime}$ or $\alpha$ for polyethylenes crystallized at $130^{\circ} \mathrm{C}$ assuming homogeneous nucleation. The value $\alpha$ is calculated according to either $E q 16$ or Eq 17. 


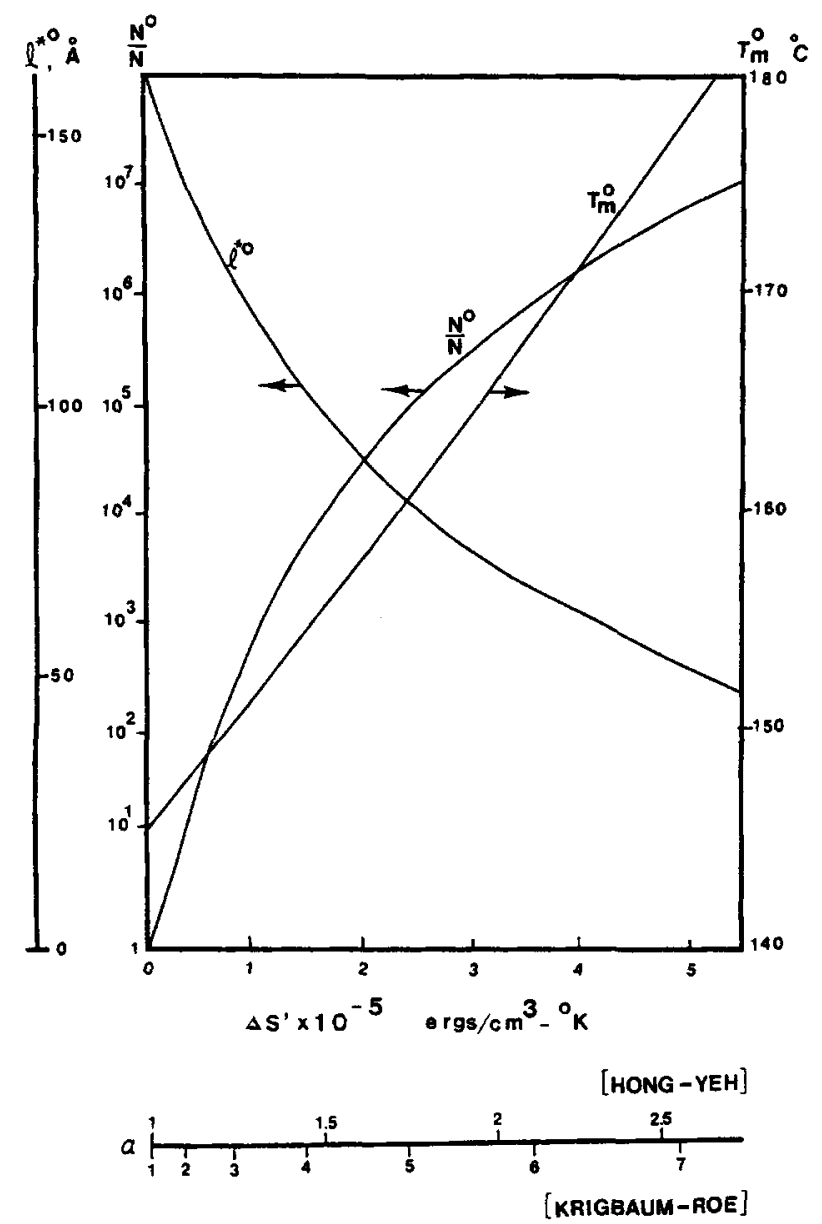

Fig. 3. Theoretical plots of $N^{\circ} / N, l^{* o}$ and $T_{m}^{\circ}$ vs $\Delta S^{\prime}$ or $\alpha$ for polyethylenes crystallized at $130^{\circ} \mathrm{C}$ assuming heterogeneous nucleation. Same constants as in Fig. 2.

is $4000(34))$, and $m \cong 122$ statistical segments/network chain, with $S=2.35$ chain segments per statistical segment (35). Of particular interest is the tremendous increase in the homogeneous nucleation rate of SIC over that of QC (Fig. 2). If both nucleation processes are assumed to be heterogeneous, the SIC rates can still be several orders of magnitude higher than QC (Fig. 3). It will be shown in Part IV (26) that comparisons with measured nucleation rates and melting temperatures will tend to suggest that the crystallization process for SIC, as in the case of QC, is heterogeneous in nature. On the other hand, since the extent of $T_{m}^{o}$ increase is independent of the type of nucleation process, accurate determinations of $T_{m}^{o}$ from $T_{o b s}^{o}$ (using $E q 13$ or the well-known Hoffman and Weeks' method for determining $T_{m}^{o}(36)$ ) can also be very useful in assessing the type of crystallization process involved, whether it be homogeneous or heterogeneous, based on such theoretical plots shown in Figs. 2 and 3.

The enhancement of nucleation rates is primarily due to two factors, namely the decrease in critical size nucleus $\left(l^{* o}\right.$ and $\left.\nu a^{* o}\right)$ and the decrease in free energy required for the formation of a critical nucleus, $\Delta F^{* 0}$. The former is thermodynamically favorable for the increase of number of nuclei, leading therefore to finer crystallites, whereas the latter is kinetically favorable for the rate of formation of nuclei.
Another important prediction is the decrease of $l^{* o}$ with increasing molecular extension, $\alpha$, or melt entropy decrease, $\Delta S^{\prime}$, or with $T_{m}^{o} / \Delta T$ according to $E q 12^{\prime}$. This is somewhat expected since our nucleation theory is based on the classical behavior of critical nucleus size variation with $1 / \Delta T$. Now we can compare the variation of the observed crystal thickness, $l_{o b s}^{o}$, or long period, $L_{o b s}^{o}$, with increasing strains or strain rates with the variation of $l * 0$ shown in Figs. 2 and 3. As pointed out in the Introduction, decrease in crystal thickness with increasing strains or strain rate has been found for several polymers, for example, for natural rubber $\left(l_{o b s}^{o} \sim 130 \AA\right.$ at $7 \times v s$ $l_{\text {calculated }} \sim 350 \AA$ at near $T_{m}$ or about $\left.25^{\circ} \mathrm{C}(5)\right)$, PET $\left(L_{\text {obs }}^{o}\right.$ $=90 \AA$ at $4 \times(10)$ vs $L_{\text {obs }}=121 \AA$ at $\left.144^{\circ} \mathrm{C}(37)\right)$, transpolyisoprene $\left(L_{o b s}^{o}=180 \AA\right.$ at $7 \times$ and at $60^{\circ} \mathrm{C}$, compared with $L_{o b s}^{o}=210 \AA$ at $7 \times$ vs $260 \AA$ at $5 \times$ vs 280 $\AA$ at $3 \times$, all at $\left.124^{\circ} \mathrm{C}(9)\right)$ and stirrer-crystallized polyethylene $\left(L_{o b s}^{o}=231 \AA\right.$ at $200 \mathrm{rpm}$ and $96.1^{\circ} \mathrm{C}$, vs $L_{o b s}^{o}=$ $189 \AA$ at $1200 \mathrm{rpm}$ and $\left.96^{\circ} \mathrm{C}(21)\right)$. These results indicate that the observed crystal thickness or long period is directly related to the critical nucleus thickness, suggesting that it is primarily a nucleation controlled process. In the time available for crystallization the defects in the form of folds do not have time to diffuse out of the crystal surface to allow growth of thicker or more extended chain crystals.

In Figs. 2 and 3, we have also included the predicted changes in "coil" dimensions based either on the assumption that rubber elasticity applies (i.e., using $E q$ 16) or using a more accurate relation between $\Delta S^{\prime}$ and $\alpha$ (Eq 17). Our analysis showed that, using $E q 17$, at entropy reductions of up to only $1.5 \times 10^{5} \mathrm{ergs} / \mathrm{cm}^{3}-{ }^{\circ} \mathrm{K}$, where substantial changes in $N^{o} / N$ and $T_{m}^{o}$ are observed, the predicted increase in molecular coil dimension is less than about 50 percent. This indicates a surprisingly low degree of chain extension actually occurring prior to SIC. It can explain why, for example, extended chain crystals are extremely hard to get by means of SIC, unless one can get complete chain extension in the amorphous state prior to SIC.

In Fig. 4, the theoretical dependencies of $N^{\circ} / N$ on $\Delta S^{\prime}, \alpha$ and $\Delta n$ (birefringence) are shown for polyethylene crystallized at four different temperatures. We have also included the results obtained by Kobayashi and Nagasawa (24). They have derived a set of very similar equations for nucleation and growth rates. According to them, their theory was applicable only to the lamellar overgrowth with chain folding occurring during the crystallization process as in the case for lamellar crystallization in the quiescent state (23). The constants chosen in Fig. 4 are the same as those used by Kobayashi and Nagasawa for direct comparison. In theory then, we should get the exact same plots in spite of the fact that the assumptions or the theoretical justifications for the two theories are entirely different. However, Fig. 4 shows that their plots do not superpose with ours, which we believe is caused by some numerical errors in their calculations.

We have also included on the abscissa their birefringence $(\Delta n)$ values which they have obtained using the $\alpha$ values obtained from $E q 16$ and some well-known 


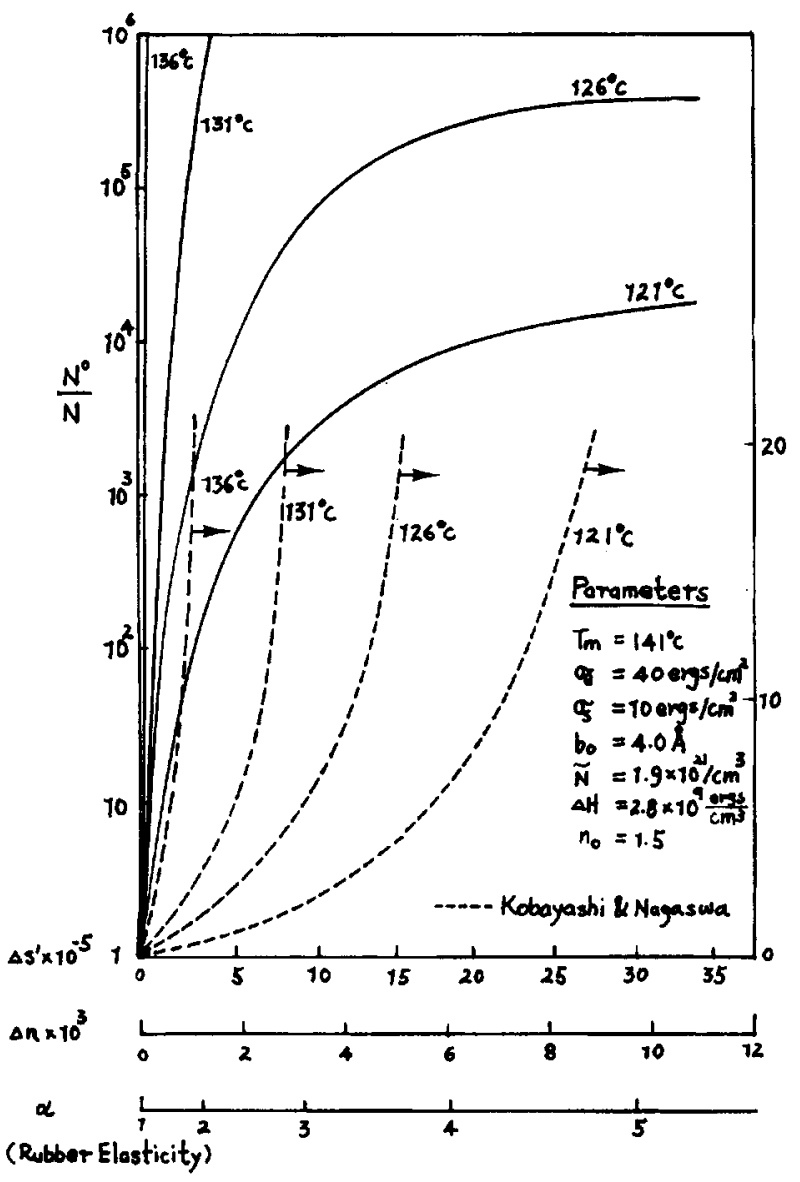

Fig. 4. Heterogeneous nucleation rate enhancements, $N^{\circ} / N$, as a function of $\Delta S^{\prime}, \Delta$ or $\alpha, \alpha$ is calculated according to Eq 16. Plots obtained by Kobayashi and Nagasawa (24) are included for comparison. The constants used are given as shown.

relationships between $\alpha$ and $\Delta n$ (35). The purpose is to show that unless a proper equation is used to obtain the value of $\alpha$, any functional relationships between $N^{\circ} / N$, $l^{* o}$, etc. and $\Delta n$ may not be accurate.

To test the present theory, it is desirable either to evaluate $\Delta S^{\prime}$ through measured birefringence or to test the fitness of $\Delta S^{\prime}$ calculated from measured $N^{\circ} / N, l^{* 0}$, $T_{m}^{o}$ or $\alpha$ under identical crystallization conditions. Experimentally, $N^{\circ} / N$ can be determined from induction time measurements to be described in Part IV (26), $l_{o b s}^{o}$ from small-ange $\mathrm{X}$-ray scattering or electron microscopy, $T_{o b s}^{o}$ (to find $T_{m}^{o}$ ) by differential scanning calorimetry and $\alpha$ by elongation ratio in the case of stretched polymer networks.

\section{REFERENCES}

1. G. S. Y. Yeh, Polym. Eng. Sci. 16, 138 (1976); ibid., 16, 145 (1976) and references therein.

2. J. Gough, Proc. Lit. Phil. Soc., Manchester, 1, 288 (1805).

3. J. R. Katz, Naturw., 4, 169 (1925); Trans. Faraday Soc., 32, 77 (1936).

4. P. J. Flory, J. Chem. Phys., 15, 397 (1947).
5. D. Luch and G. S. Y. Yeh, J. Appl. Phys., 43, 4326 (1972).

6. D. Luch and G. S. Y. Yeh, J. Macromol. Sci.-Phys., B7, 121 (1973).

7. D. Luch and G. S. Y. Yeh, J. Polym. Sci., 11, 467 (1973).

8. I. R. Hardin and G. S. Y. Yeh, J. Macromol. Sci.-Phys., B7, 375 (1973).

9. I. R. Hardin and G. S. Y. Yeh, ibid., B7, 393 (1973).

10. G. S. Y. Yeh and P. H. Geil, ibid., B1, 251 (1967).

11. S. L. Lambert and G. S. Y. Yeh, J. Appl. Phys., 42, 4614 (1971).

12. D. L. Krueger and G. S. Y. Yeh, J. Macromol. Sci.-Phys., B6, 431 (1972).

13. D. L. Krueger and G. S. Y. Yeh, J. Appl. Phys., 43, 4339 (1972).

14. A. Keller and M. J. Machin, J. Macromol. Sci.-Phys., B1, 41 (1967).

15. A. J. Pennings, Kolloid Z., 237, 336 (1970).

16. A. Keller and M. J. Mackley, Pure Appl. Chem., 39, 195 (1974).

17. T. Nagasawa and Y. Shimomura, J. Polym Sci. Polym. Phys. Ed., 12, 2291 (1974).

18. A. J. Pennings, 5th Europhysics Conference, Abstracts on Orientation Effects in Solid Polymers, p. 164 (1976).

19. M. Kapuscinski, I. M. Ward, and J. Scanlan, J. Macromol. Sci.-Phys., B11, 475 (1975).

20. J. A. Odell, D. A. Grubb, and A. Keller, Polym. Prepr., 19, 331 (1978).

21. A. Keller and F. M. Willmouth, J. Macromol. Sci.-Phys., B6, 493 (1972).

22. T. Kawai, M. Iguchi, and H. Tonami, Kolloid Z., 221, 28 (1967).

23. J. K. Lauritzen, Jr. and J. D. Hoffman, J. Res. NBS, 64A, 73 (1960).

24. K. Kobayashi and T. Nagasawa,J. Macromol. Sci.Phys., B4, $331(1970)$.

25. G. S. Y. Yeh, J. Macromol. Sci.-Phys., B6, 465 (1972)

26. G. S. Y. Yeh, K. Z. Hong, and D. L. Krueger, Polym. Eng. Sci., 19, 401 (1979).

27. K. Z. Hong, and G. S. Y. Yeh, "Strain-Induced Crystallization: Part V: Melting Behavior," to be published.

28. W. R. Krigbaum and R. J. Roe, J. Polym. Sci., Part A, 2, 4391 (1964).

29. D. Turnbull and J. C. Fisher, J. Chem. Phys., 17, 71 (1949).

30. F. P. Price, in "Nucleation," ed. by A. C. Zettlemoyer, Marcel-Dekker, N.Y., (1969).

31. See, for example, J. Schultz, in "Polymer Materials Science," p. 295, Prentice-Hall, N.J. (1974).

32. J. D. Hoffman, G. T. Davis, and J. I. Lauritzen, Jr., "The Rate of Crystallization of Linear Polymers with Chain Folding," in "Treatise on Solid State Chem.," vol. 3, ed. by N. B. Hannay, Plenum Press, N.Y. (1976).

33. P. H. Geil, "Polymer Single Crystals," Interscience, N.Y. (1963).

34. R. S. Porter, W. J. MacKnight, and J. F. Johnson, Rubber Chem. Technol., 40, 1 (1967).

35. L. R. G. Treloar, "The Physics of Rubber Elasticity," p. 102, Oxford (1949).

36. J. D. Hoffman and J. J. Weeks, J. Res. NBS., 66A, 13(1962).

37. G. S. Y. Yeh and P. H. Geil, J. Macromol. Sci.-Phys., B1, 235 (1967).

38. W. R. Krigbaum, J. V. Dawkins, G. H. Via, and Y. I. Balta, J. Polym. Sci., Part A-2, 4, 475 (1966).

39. F. de Candia, G. Romano, and V. Vittoria, J. Polym. Sci. Polym. Phys. Ed., 11, 2291 (1973).

40. R. J. Samuels, J. Polym. Sci., Polym. Phys. Ed., 13, 1417 (1975).

41. M. Todki and T. Kawaguchi, J. Polym. Sci., Polym. Phys. Ed., 15, 1507 (1977). 